\title{
Das therapeutische Ergebnis komplementär- medizinischer Anwendungen kann wissenschaftlich nicht gemessen werden. Stimmt das?
}

\author{
R. H. Adler, C. E. Minder
}

\section{Wo findet Wissenschaft statt?}

Nach Odegaard [1] besteht Wissenschaft darin, mit höchstem denkerischem Bemühen Regeln für Beobachtungen zu entwickeln und diese Regeln an weiteren Beobachtungen zu bestätigen. Bedeutsam an dieser Definition ist, dass sie kein Gebiet der Wissenschaft zuordnet oder von ihr ausschliesst.

Damit ist die Komplementärmedizin (KM) weder wissenschaftlich noch unwissenschaftlich. Erst die Art und Weise des Umgangs mit ihr entscheidet, ob sie wissenschaftlich oder unwissenschaftlich ist, und ob ihre Ergebnisse wissenschaftlicher Prüfung standhalten. Die gängigen Theorien der Wissenschaftlichkeit unterscheiden sich im übrigen kaum. Dies heisst aber nicht, dass es je nach Gebiet keine unterschiedlichen Schwierigkeiten gibt, die allgemeinen Prinzipien der Wissenschaft anzuwenden.

\section{Harte und weiche Daten oder Zeichen}

Die KM möchte wie die Schulmedizin (SM) Symptome lindern oder beheben und Krankheiten mildern oder heilen. Krankheitszeichen wie die eingeschränkte glomeruläre Clearance werden durch Messen des Kreatininspiegels im Serum und der Quantität des im Urin ausgeschiedenen Kreatinins erfasst. Für die Messung dieser Werte braucht es keine Interaktion zwischen Arzt und Patient, die Werte können wiederholt gemessen werden und sind in Referenzlabors kontrollierbar. Das Kreatinin ist lediglich eine Funktion der Muskelmasse und der glomerulären Filtration. Charakteristika dieser Zeichen sind ihre Abhängigkeit von nur wenigen Faktoren und ihre Unabhängigkeit von der Arzt-Patienten-Beziehung. Solche Zeichen werden oft als harte Daten bezeichnet und stellen das Forschungsgebiet der Biomedizin dar, wie sie sich seit dem 17. Jahrhundert entwickelt hat [2]. Zeichen wie Schmerz,
Durst, Angst und Depression sind durch mehrere Faktoren bestimmt. Für ihre Erfassung braucht es das Gespräch zwischen Arzt und Patient. Der Patient stellt dabei ein geschlossenes System dar [3], das sich nur der Empathie des Arztes öffnet und die privaten Empfindungen preisgibt. Solche Zeichen werden oft als weich bezeichnet. Sie sind weder weniger noch mehr wert als harte Zeichen [4]. Es kommt auf die Fragestellung an. Die Bewegung der Hände und Arme mit supinierten Handflächen nach oben und das anschliessende Fallenlassen der Arme mit Pronation der Hände ist ein weiches Zeichen und drückt meist Hoffnungslosigkeit und Resignation aus [5]. Wird es vom Arzt verpasst, geschieht vielleicht ein verhinderbarer Suizid. Somit ist ein weiches Zeichen in gewissen Umständen wichtig, ein vorübergehend erhöhtes Serumkreatinin als hartes Zeichen vielleicht weniger bedeutsam.

Die moderne SM, die sich zur biopsychosozialen Medizin entwickelt hat [6], befasst sich mit harten und weichen Zeichen. Die KM behauptet oft, sie sei mit wissenschaftlichen Methoden der SM nicht erfassbar, weil Zeichen, für die sie sich interessiert (im obigen Sinn weiche Zeichen), nicht mit den üblichen SM-Methoden erfassbar seien. Messen bedeutet immer auch Deuten. Weiche Daten zu deuten ist schwieriger, als harte Daten zu interpretieren, weil sie meistens mehr determinierende Faktoren aufweisen als harte. Aber auch in «harten» Wissenschaften, etwa der Physik, kann das Interpretieren problematisch sein.

Die Sozialwissenschaften und die Psychologie stellen Bewertungsverfahren bereit, mit denen Symptome wie Gestimmtheit, Schmerz, Angst, Depression und Hoffnungslosigkeit messbar werden. Als Zugang gibt es Interviews, Fragebogen, verbale und visuale Ratingskalen. Im Gebiet des Schmerzes erlaubt z.B. die VisualAnalog-Skala, Dosis-Wirkungs-Kurven zu erstellen. Bei den Skalen handelt es sich oft um solche, die «klein, grösser, noch grösser ...» erfassen. Sie 
heissen Ordinalskalen. Mit ihnen lässt sich ebenso gut arbeiten wie mit Skalen, die von Stufe zu Stufe gleiche Abstände aufweisen, Intervallskalen, oder sogar noch einen Nullpunkt haben, die Rationalskalen. Letztere werden oft für harte Daten eingesetzt. Nota bene: Es gibt weder für die SM spezifische Hoffnungslosigkeit oder Erschöpfung noch KM-typische. Hoffnungslosigkeit ist Hoffnungslosigkeit, und Erschöpfung ist Erschöpfung, auch wenn sie in der Wirklichkeit des einzelnen unterschiedlich erlebt werden.

Somit ist das Argument der KM, ihre Therapieergebnisse seien nicht mit Methoden zu erfassen, die in der SM üblich sind, hinfällig.

\section{KM ist ganzheitlich und damit prinzipiell unterschiedlich zur SM}

Der Begriff «ganzheitlich» muss definierbar sein, damit er der Medizin dienlich werden kann [7]. Ohne Definition behindert er die Suche nach Strukturen und Funktionen, die den lebenden Organismus verstehen lassen. Bisher hat die KM keine einleuchtende holistische Theorie vorgelegt. Die Behauptung, eine KM-Anwendung beeinflusse im Gegensatz zur SM eben den ganzen Organismus, stimmt nicht. Die SM beeinflusst auch den Gesamtorganismus, denn die Einwirkung eines einzigen Faktors auf ein System vermag dieses System als ganzes zu verändern. Schwemmt ein Diuretikum Ödeme aus, so vermindert sich die kardiale Vorlast, die Pumpleistung verbessert sich, damit die Oxygenierung des Gehirns, die zu einer verbesserten Funktion des psychischen Apparates führt, die wiederum eine günstigere Verarbeitung von Stress erlaubt usw. Der reflektierte Reduktionismus in der Forschung ist unentbehrlich, das Messenwollen einer Ganzheitlichkeit illusorisch. Von «ganzheitlich» kann erst gesprochen werden, wenn die ganzheitliche Theorie erklärt, wie die hierarchischen Ebenen im lebenden Organismus gegenseitig verbunden sind, und wie dieser Organismus mit seiner Umwelt vernetzt ist [3]. Wird z. B. Stimmung erfasst, so stellt diese eine willkürliche Auslese aus der Vielfalt der Affekte dar und entspricht nicht der Wahrnehmung und Messung einer Ganzheit.

\section{Randomisierte Studien als Paradigma der SM für die KM nicht anwendbar}

Sowohl beim Verumarm als auch beim Placeboarm einer Studie ist der Experimentator/Arzt als Therapeut beteiligt. Die Gefühle des Patienten wie Angst oder Durchhaltewille, Einstellung zur Therapieform und zum Therapeuten/Experimentator können gemessen werden, indem ihm mittels Interview oder Fragebogen die Gelegenheit gegeben wird, sich auszudrücken. Das gleiche ist für den Experimentator/therapierenden Arzt möglich. Es kann erfasst werden, ob er sich beteiligt fühlt, ob er der Meinung ist, dass sein Verum ohne sein Wissen, dass es ein Verum sei, nicht wirksam sein könne. Ist er beispielsweise dieser Meinung, so wird sich der Einfluss seiner Haltung bei Placebo- und Verumarm nicht unterscheiden. Ist er jedoch überzeugt, dass seine positive Einstellung für die Verumwirksamkeit nötig ist und zeigt auch der Placeboarm die Wirkung, dann heisst dies, dass das Verum nicht wirksamer ist als das Placebo. Wären alle Patienten gleich, könnten auf beliebige Weise zwei völlig vergleichbare Gruppen hergestellt werden. In Wissensbereichen ohne quantitativ bedeutsame interindividuelle Variabilität, wie z. B. in weiten Gebieten der Physik und Chemie, wird das auch so gehandhabt. In Wissensbereichen, bei denen die nicht kontrollierbare Variabilität quantitativ wichtig ist, ist heute nur eine Weise bekannt, in der zwei vergleichbare Gruppen geformt werden können: die Randomisierung. Ihr Prinzip zur Schaffung vergleichbarer Gruppen wurde übrigens auch in der schulmedizinischen Forschung nur zögernd und mit grosser Verspätung übernommen. Es wurde zuerst in landwirtschaftlichen Versuchen angewendet und geht auf den Genetiker und Statistiker R. A. Fisher zurück.

\section{Schlussfolgerung}

Die dargelegten Argumente sollten genügen, um zu zeigen, dass die SM nicht willkürlich definiert, was wissenschaftlich ist. Ein konstruktiver Beitrag der KM wäre, an der Weiterentwicklung und Anpassung der Forschungsmethoden zu arbeiten, statt die Messung von Beobachtungen als unannehmbar für die KM auszuschlagen. So sind beispielsweise durchaus Untersuchungen in Praxen möglich, etwa bei homöopathischen Anwendungen, und es ist unverständlich, warum das ärztliche Vorgehen in einer Praxis in wissenschaftlich guten Studien angeblich nicht berücksichtigt werden könne.

Noch ein Gedanke zur Zulassungsfrage zur Grundversorgung: Sollen Gebiete wie Chinesische Medizin, Anthroposophische Medizin usw. zum Katalog der Grundversorgung zugelassen werden? Uns scheint die Frage falsch gestellt. Analog zur Chirurgie, die auch nicht als Ganzes zugelassen ist, sollten die einzelnen An- 
wendungen geprüft werden. Beispielsweise sind Schönheitsoperationen im allgemeinen nicht zugelassen, die Verkleinerung einer zu grossen Brustmasse, die statische Probleme mit Krankheitswert zur Folge hat, jedoch schon. Es sollte wie bei den Medikamenten der Pharmafirmen vorgegangen werden. Dort werden nicht einfach Medikamente zugelassen oder abgelehnt, sondern die Zulassung erfolgt jeweils für spezifische Indikationen.

\section{Literatur}

1 Odegaard CE. Dear Doctor. A personal letter to a physician. Menlo Park, CA: The Henry J Kaiser Family Foundation Found; 1986.

2 Engel GL. How much longer must medicine's science be bound by a $17^{\text {th }}$ century world view? In: White KL (ed.). The Task of Medicine. Dialog at Wickenburg. Menlo Park, CA: The Henry J Kaiser Family Foundation; 1988. p. 113-36.

3 von Uexküll T, Wesiack W. Integrierte Medizin als Konzept der Heilkunde: Ein bio-psychosoziales Modell. Kap. 1. 3-42. In: von Uexküll T. Psychosomatische Medizin. 6. Auflage. Herausgegeben von Adler RH et al. München und Jena: Urban und Fischer; 2003.

4 Adler RH. Hard and soft data. A semiotic point of view. Schweiz Med Wochenschr 2000;130: 1249-51.

5 Schmale AH Jr, Engel GL. The giving-up, given-up complex on film. Arch Gen Psychiat 1967;17: $135-45$.

6 Foss L, Rothenberg K. The Second Medical Revolution. From Biomedicine to Infomedicine. Shambala, Boston, London: New Science Library; 1987.

7 Adler RH. Ganzheitlich - Der Begriff, sein Missbrauch und einige Klärungen. Schweiz Med Wochenschr 1998;128:1075-8. 